\title{
Thymic stromal lymphopoietin (TSLP)-mediated dermal inflammation aggravates experimental asthma
}

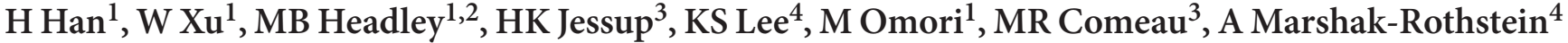 \\ and SF Ziegler ${ }^{1,2}$
}

Individuals with one atopic disease are far more likely to develop a second. Approximately half of all atopic dermatitis (AD) patients subsequently develop asthma, particularly those with severe AD. This association, suggesting a role for $A D$ as an entry point for subsequent allergic disease, is a phenomenon known as the "atopic march." Although the underlying cause of the atopic march remains unknown, recent evidence suggests a role for the cytokine thymic stromal lymphopoietin (TSLP). We have established a mouse model to determine whether TSLP plays a role in this phenomenon, and in this study show that mice exposed to the antigen ovalbumin (OVA) in the skin in the presence of TSLP develop severe airway inflammation when later challenged with the same antigen in the lung. Interestingly, neither TSLP production in the lung nor circulating TSLP is required to aggravate the asthma that was induced upon subsequent antigen challenge. However, CD4 T cells are required in the challenge phase of the response, as was challenge with the sensitizing antigen, demonstrating that the response was antigen specific. This study, which provides a clean mouse model to study human atopic march, indicates that skin-derived TSLP may represent an important factor that triggers progression from $A D$ to asthma.

\section{INTRODUCTION}

Atopic dermatitis $(\mathrm{AD})$, one of the most common allergic skin inflammatory diseases, has a particularly high prevalence among infants and children. Onset is during the first 6 months of life in $45 \%$ of all cases. ${ }^{1}$ Based on several longitudinal studies, approximately half of AD patients will develop asthma, especially with severe $\mathrm{AD}$, and two-thirds will develop allergic rhinitis. In general, the clinical signs of $\mathrm{AD}$ predate the development of asthma and allergic rhinitis, suggesting that $\mathrm{AD}$ is an entry point for subsequent allergic diseases, ${ }^{2,3}$ but the molecular mechanisms underlying the "atopic march" remain unclear.

Recently, thymic stromal lymphopoietin (TSLP), an interleukin-7 (IL-7)-related cytokine, has emerged as an important potential contributor to allergic inflammation. Although TSLP was initially identified and characterized in thymic stromal cells, ${ }^{4,5}$ it is expressed predominantly in epithelial cells (ECs) of the skin (keratinocytes), lung (small airway ECs), and intestine (intestinal ECs). ${ }^{6-8}$ Originally discovered because of its potent effects on B- and T-cell growth and differentiation, ${ }^{9-11}$ TSLP has also been shown to be associated with the pathogenesis of multiple inflammatory diseases including $\mathrm{AD}$ and asthma. The involvement of TSLP in allergic inflammation was first demonstrated in $\mathrm{AD}$ patients. TSLP protein, although undetectable in normal and nonlesional skin in these patients, is highly expressed in acute and chronic AD lesions. ${ }^{8}$ In addition, human asthmatics have increased concentrations of TSLP in their airways correlating with T helper type 2 (Th2)-attracting chemokine expression and disease severity. ${ }^{12}$ Murine models have extended these findings, showing that inducible epidermal TSLP expression induces a spontaneous AD-like disease ${ }^{13}$ characterized by all the hallmark features of human $\mathrm{AD}$. In contrast, TSLP receptor-deficient mice exhibited a dramatically reduced Th2-type inflammatory response in a fluorescein isothiocyanate contact hypersensitivity-based model of AD. ${ }^{14}$ Mice expressing surfactant protein $\mathrm{C}$ promoter-driven TSLP in the lung developed severe airway inflammation, ${ }^{15}$ whereas mice deficient

${ }^{1}$ Immunology Program, Benaroya Research Institute, Seattle, Washington, USA. ${ }^{2}$ Department of Immunology, University of Washington School of Medicine, Seattle, Washington, USA. ${ }^{3}$ Inflammation Research, Amgen, Seattle, Washington, USA. ${ }^{4}$ Department of Medicine, Division of Rheumatology, University of Massachusetts Medical School, Worcester, Massachusetts, USA. Correspondence: SF Ziegler (sziegler@benaroyaresearch.org)

Received 14 September 2011; accepted 8 January 2012; published online 22 February 2012. doi:10.1038/mi.2012.14 
in the TSLP receptor failed to develop asthma in response to inhaled antigen..$^{15,16}$

TSLP is considered a likely candidate for perpetuating the atopic march for several reasons. First, TSLP expression has been found at elevated levels in the skin of $\mathrm{AD}$ patients and in the lungs of asthmatics. ${ }^{8} 12$ Second, TSLP is an EC-derived cytokine; and both $\mathrm{AD}$ and asthma develop at EC surfaces. ${ }^{17}$ Third, as described previously, a role for TSLP in allergic inflammation has been demonstrated in both humans and mice. Finally, three models of induced expression of TSLP in mouse keratinocytes resulted in subsequent airway inflammation following challenge. These models include topical application of a vitamin D3 ana$\log$ and keratinocyte-specific deletion of the Notch signaling pathway. ${ }^{18,19}$ An important caveat in these studies is that the methods used to induce TSLP expression results in artificially high systemic levels of TSLP, which is not seen in AD patients.

To develop a model to test the role of TSLP in the atopic march, we extended work showing that intradermal injection of TSLP in wild-type mice led to a marked increase of inflammatory infiltrate in the subcutis, in the absence of detectable circulating cytokine. ${ }^{20}$ We administered TSLP, in the presence of antigen, via intradermal injection, to ask whether subsequent challenge with antigen in lung was capable of inducing airway inflammatory disease. We demonstrate that intradermal administration of TSLP and ovalbumin (OVA) leads to an allergic asthma-like phenotype upon intranasal challenge with OVA. Interestingly, in contrast to previous studies, ${ }^{18}$ neither TSLP production in the lung nor circulating TSLP is required to aggravate the disease induced upon OVA challenge. TSLP-induced responses are antigen specific and T-cell dependent in this model. In addition, allergic disease development is blocked through oral exposure to OVA antigen before, but not after, skin sensitization. Our study demonstrates that skin-restricted TSLP triggers a progression from $\mathrm{AD}$ to asthma.

\section{RESULTS}

Mice sensitized in the skin in the presence of TSLP develop aggravated asthma-like airway inflammation upon challenge

Epidemiological studies have shown that children with AD, especially those with severe disease, are likely to also develop asthma, demonstrating a link between these two conditions. ${ }^{2}$ Given its purported role in these allergic diseases, TSLP was a likely candidate as the factor that links $\mathrm{AD}$ and asthma. Our laboratory has found that mice with a skin-specific inducible TSLP transgene develop an AD-like disease, as well as pulmonary inflammation (see ref. 13 and Supplementary Figure S1 online). However, we also observed inflammatory infiltrates in a wide variety of organs including liver, gastrointestinal tract, and spleen (Supplementary Figure S1 online) perhaps because of elevated systemic levels of TSLP. We therefore sought to develop a mouse model that recapitulates human atopic disease and determine whether TSLP plays a role in this phenomenon. To test this, we modified a protocol from Jessup et al. ${ }^{20}$ for TSLPmediated skin inflammation. Wild-type BALB/c mice were treated with TSLP and the antigen OVA intradermally (four times over 2 weeks) to induce an $\mathrm{AD}$-like skin inflammatory disease. Following a 9-day rest period, the mice were treated intranasally with OVA for four consecutive days (Figure 1a). Consistent with the induction of Th2-type inflammation by TSLP treatment, total serum immunoglobulin E (IgE) and OVAspecific IgE were markedly elevated in TSLP + OVA-treated mice (Figure 1b,c). To assess lung infiltration of leukocytes, bronchoalveolar lavage (BAL) fluid was analyzed on day 25 (D25). In phosphate-buffered saline-exposed mice, regardless of sensitization status, the predominant cell type seen in BAL was the alveolar macrophage. TSLP skin sensitization without antigen failed to alter the cellular composition of the BAL upon subsequent OVA re-challenge, whereas mice where mouse serum albumin (MSA) was substituted for TSLP during the sensitization phase showed only mildly increased cell infiltration. In contrast, following OVA re-challenge, exposure of mice to TSLP + OVA at sensitization caused an influx of eosinophils into the BAL fluid and increased cellular infiltrate threefold over the mice given MSA + OVA (Figure 1d).

Consistent with increases in BAL cellularity, histopathologi$\mathrm{cal}$ analysis of lung section showed that only mice sensitized in the skin with TSLP + OVA showed severe inflammatory infiltrates (data not shown). In addition, lungs from these mice also showed enhanced goblet cell metaplasia and mucus overproduction by periodic acid-Schiff staining, whereas lungs from the other groups did not (Figure 1e). This suggests that intradermal administration of TSLP during the antigen sensitization phase is necessary to aggravate allergic airway inflammation in this model.

IL-4, IL-5, IL-10, and IL-13 production from restimulated inguinal lymph node $\mathrm{CD} 4 \mathrm{~T}$ cells was very low and showed no difference compared with control groups, indicating that skin inflammation was resolved at the time that lung inflammation developed (data not shown). Analysis of lung cytokine RNA levels showed that, compared with MSA + OVA treatment, TSLP + OVA treatment induced the production of the Th2 cytokines IL-4, IL-10, IL-13, and tumor necrosis factor, as well as of eosinophil chemoattractant chemokine (eotaxin-2; Figure 2a).

To ensure that the disease we observed in this model system was TSLP dependent and not because of the influence of potential contaminating factors such as lipopolysaccharide in either the TSLP or OVA stocks, lipopolysaccharide-unresponsive TLR $4^{\text {mut }}$ mice were subjected to the same skin sensitization and lung challenge model. The TLR4 ${ }^{\mathrm{mut}}$ mice developed disease indistinguishable from that seen in wild-type mice (Supplementary Figure S2 online). Thus, the aggravated airway inflammatory response seen in wild-type mice was because of the presence of TSLP during the sensitization step.

To determine whether increasing the time between skin exposure to TSLP plus antigen and lung challenge affected development of airway inflammation, the interval between skin sensitization and lung challenge was increased from 9 to 30 days. The airway inflammation that developed in mice sensitized in the skin with TSLP + OVA in this protocol was indistinguishable from that shown in Figure 1 for mice rested 9 days (Supplementary Figure $\mathbf{3}$ online). 
a TSLP and OVA (intradermal sensitization)

D21-24: OVA or saline (intranasal challenge)
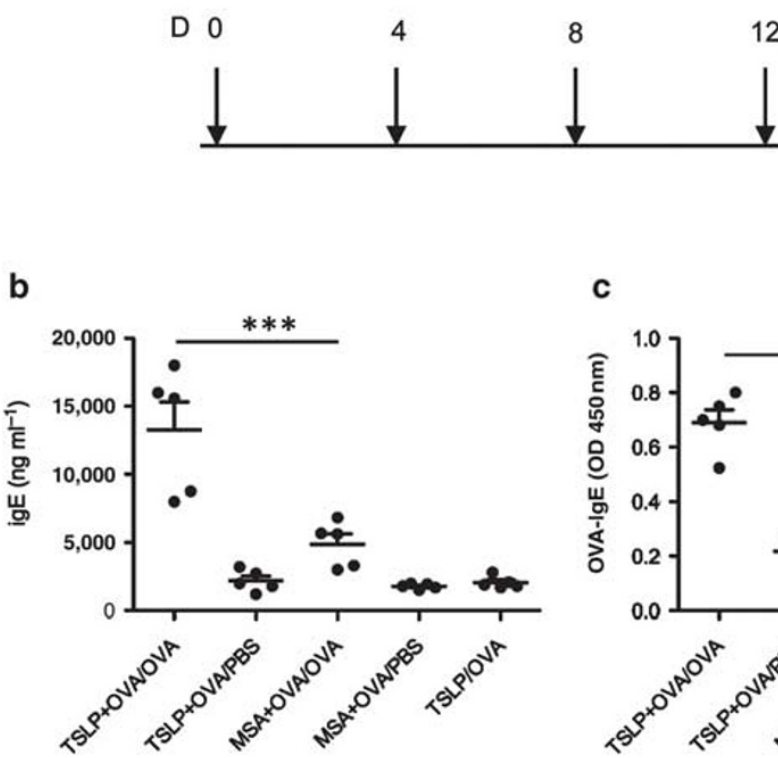

12

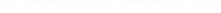

C

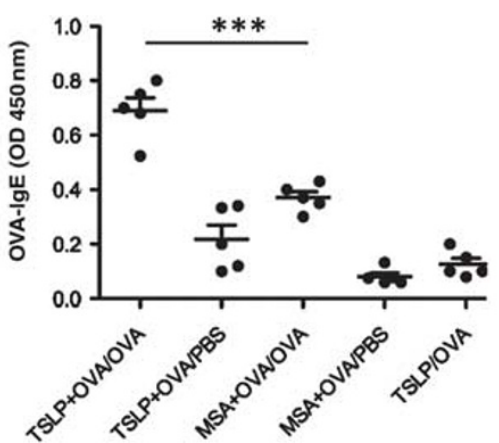

d

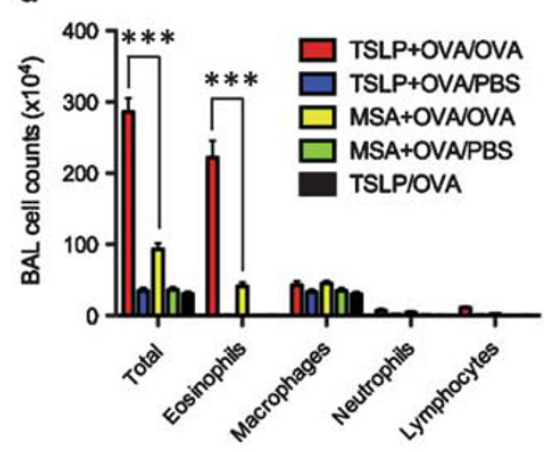

e

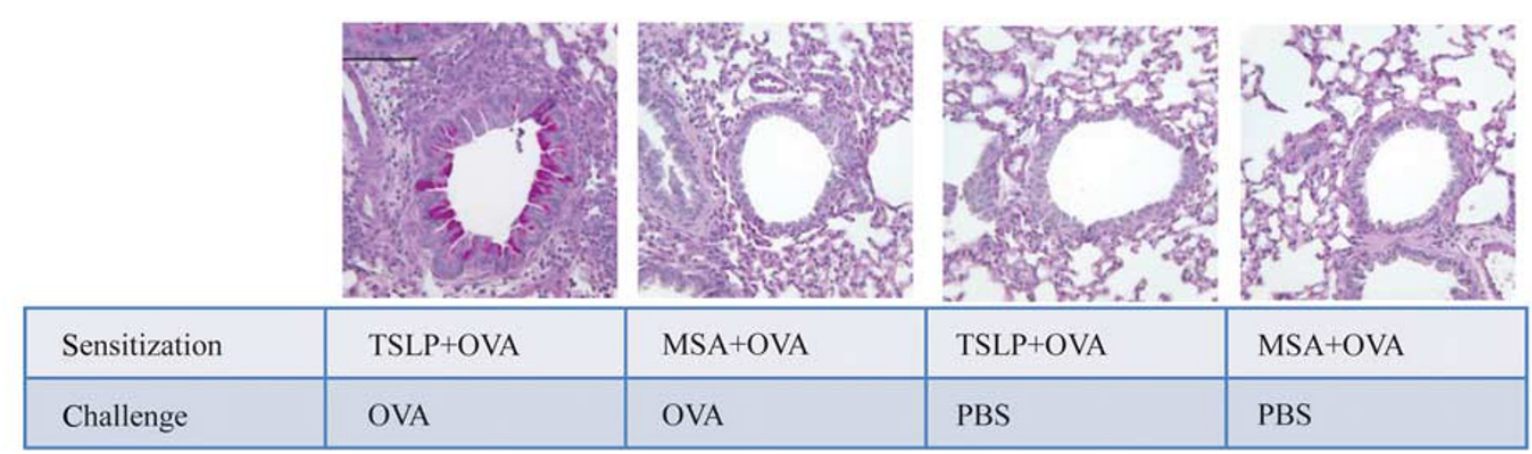

Figure 1 Intradermal administration of thymic stromal lymphopoietin (TSLP) aggravates airway inflammation and mucus secretion in experimental asthma. (a) Experimental protocol. Mice received injections of TSLP and ovalbumin (OVA; $5 \mu \mathrm{g}$ ) four times for 12 days and rested for 9 days. To induce experimental asthma, mice were OVA challenged (50 $\mu \mathrm{g}$ in $30 \mu \mathrm{l}$ saline) on 4 consecutive days (D21-D24) by intranasal instillation. Mice were killed on D25 for analysis. (b) Total serum immunoglobulin E (IgE) and (c) OVA-specific lgE on D25 ( $n=5$, two independent experiments). (d) Cell counts in the bronchoalveolar lavage (BAL) fluid. The significance between two groups was determined by two-tailed Student's $t$-test ( $n=5$, two independent experiments). (e) Representative lung tissue cross sections stained with periodic acid-Schiff (PA S) to visualize mucus-producing goblet cells and airway inflammation. MSA, mouse serum albumin; PBS, phosphate-buffered saline. Bar $=100 \mu \mathrm{m} .{ }^{\star \star \star} P \leq 0.001$.

To determine the specificity of the response to skin sensitization in the presence of TSLP, we asked whether a second epithelial-derived cytokine, IL-25, could promote a similar set of responses. Rapidly emerging studies implicate IL-25 as a critical regulator of innate and adaptive immune responses associated with Th2 cytokine-mediated inflammation at mucosal sites. ${ }^{21}$ In addition, IL-25 expression is elevated in the skin of AD patients, suggesting a role in disease. ${ }^{22}$ However, mice sensitized in the skin with IL-25 + OVA, followed by pulmonary challenge with OVA, showed no elevated levels of total serum IgE and OVAspecific IgE (Supplementary Figure S4A and B online) and mildly increased cell infiltration (Supplementary Figure S4C online).

\section{Airway inflammation does not require systemic TSLP}

The data shown above clearly show the requirement for TSLP during the skin sensitization phase in this model. To begin to determine if TSLP was also involved directly during the lung challenge, TSLP mRNA expression levels in the lungs of all treatment groups was measured on D25, as was circulating TSLP in the serum on days 14 and 25. Lung TSLP mRNA levels were equivalent in all groups (Figure 2a), and TSLP protein in the serum was undetectable $\left(<7.8 \mathrm{pg} \mathrm{ml}^{-1}\right)$, suggesting that circulating TSLP is not required for the development of allergic airway inflammation upon re-challenge in this model (Figure $\mathbf{2 b}, \mathbf{c}$ ). To further assess the role of TSLP in this process, we took two approaches. For the first approach, TSLP-deficient mice were sensitized in the skin with TSLP + OVA as before, and then challenged intranasally with OVA. As shown in Figure 3a, the TSLPdeficient mice responded to the intranasal challenge as robustly as the TSLP-sufficient mice, showing that TSLP was not required for the airway challenge. To exclude a role for systemic TSLP during the skin sensitization phase, mice were treated intranasally with an antibody to murine TSLP (anti-TSLP) on D0 and 
a
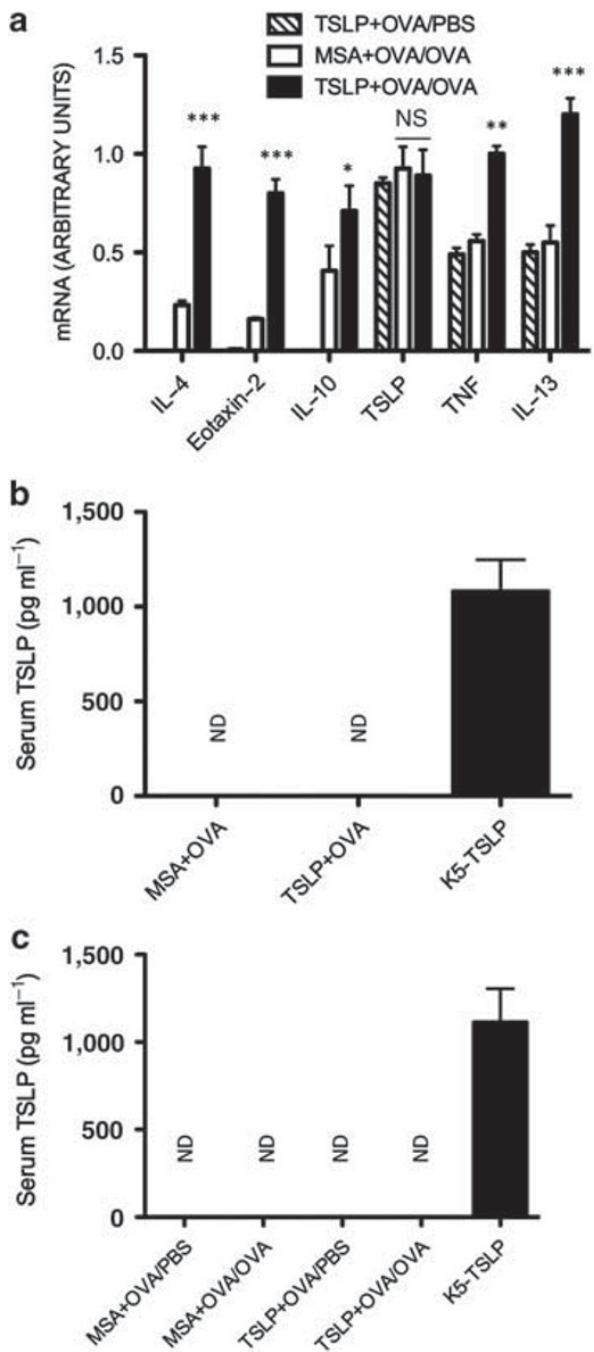

Figure 2 Airway inflammation does not require systemic thymic stromal lymphopoietin (TSLP). (a) No locally induced TSLP. Quantitative reverse transcriptase PCR (RT-PCR) analyses of cytokines and chemokines in lungs on day 25 (D25). $n \geq 4$ mice per group. The significance of gene expression between two groups was determined by two-tailed Student's $t$-test. (b and c) No circulating TSLP. Serum TSLP levels not enhanced on (b) D14 and (c) D25. K5-TSLP mice were treated on $1 \mathrm{mg} \mathrm{ml}^{-1}$ of doxycycline for 3 weeks and used as positive control. $n \geq 4$ mice per group. ND, $<7.8 \mathrm{pg} \mathrm{ml}^{-1}$. IL, interleukin; MSA, mouse serum albumin; ND, not detected; NS, not significant; OVA, ovalbumin; PBS, phosphate-buffered saline; TNF, tumor necrosis factor. ${ }^{\star} P \leq 0.05,{ }^{\star \star} P \leq 0.01$, and ${ }^{* \star \star} P \leq 0.001$.

D8. Airway inflammation in anti-TSLP-treated animals was indistinguishable from that seen in control antibody-treated mice (Supplementary Figure S5 online). To ascertain whether TSLP was required during airway challenge, TSLP + OVAsensitized mice were given anti-TSLP the days before intranasal OVA challenge. Again, anti-TSLP-treated mice developed robust airway inflammation following OVA challenge (Figure 3b). Taken together, we conclude from these studies that increased skin-restricted TSLP levels during the antigen sensitization phase is sufficient to yield an aggravated response to antigen when subsequently encountered within the lung.
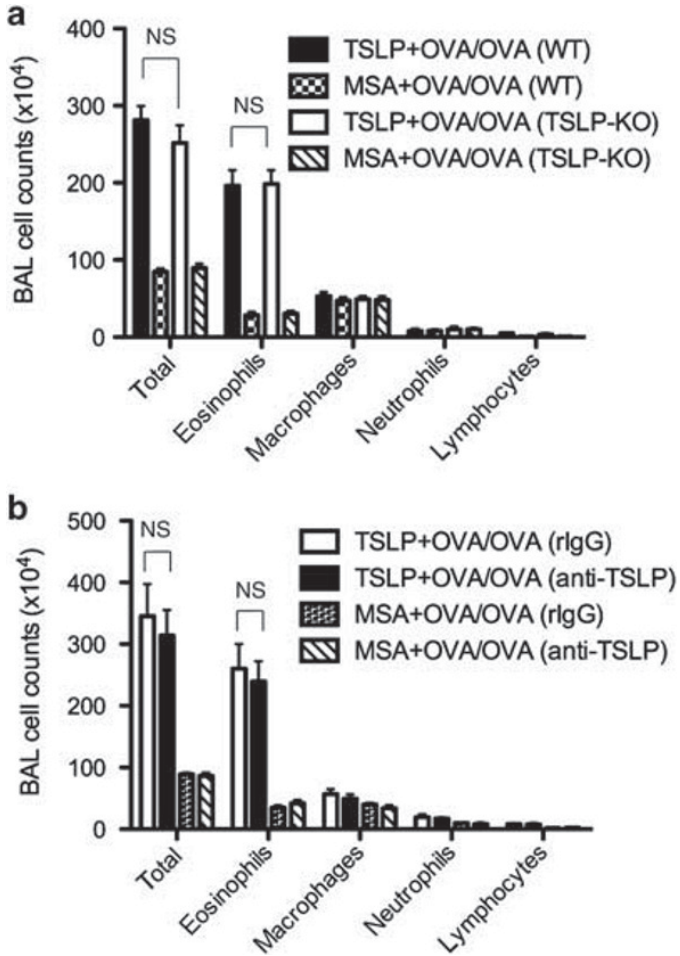

Figure 3 Thymic stromal lymphopoietin (TSLP) is dispensable once skin inflammation develops. (a) Total number and differential cell counts of cells isolated from bronchoalveolar lavage (BAL) taken from TSLPdeficient (TSLP-KO) or wild-type (WT) BALB/c mice treated with mouse serum albumin (MSA) or TSLP plus ovalbumin (OVA) followed by OVA challenge. $n \geq 5$ mice per group from two independent experiments. (b) Acute blockade of TSLP after skin sensitization does not inhibit allergic asthma. Cell counts in the BAL fluid. $n \geq 4$ mice per group from two independent experiments. The significance between two groups was determined by two-tailed Student's $t$-test. NS, not significant.

\section{Airway disease development is dependent on CD4 T cells}

Studies in mouse models of asthma have shown that allergic airway inflammation is dependent on CD4 T cells and, more specifically, is seen when T-cell receptor-transgenic Th2, but not Th1, cells are adoptively transferred to mice. ${ }^{23-27}$ TSLP, when injected intradermally together with OVA, induced IL-4producing CD $4+$ T cells (Supplementary Figure S6 online), as described before. ${ }^{20}$ The role of CD 4 T cells in atopic airway inflammation was assessed in mice treated with the depleting anti-CD4 antibody GK1.5. Mice were treated with anti-CD4 9 days before intranasal OVA challenge, and again 3 days before. This led to a nearly complete loss of CD $4+\mathrm{T}$ cells. Acute depletion of CD4 T cells also led to a dramatic reduction in airway disease development, as measured by BAL infiltrates, airway eosinophilia, and mucus production (Figure 4a and data not shown). These data demonstrate that CD $4+\mathrm{T}$ cells are required for the induction of pulmonary inflammation following skin sensitization.

To determine the nature of the CD4 T cells that were driving lung pathology, TSLP + OVA-primed mice were depleted of CD4 T cells as described above, and naive, OVA-specific DO11.10 CD4 T cells were adoptively transferred before intranasal OVA challenge. As shown in Supplementary Figure S7 

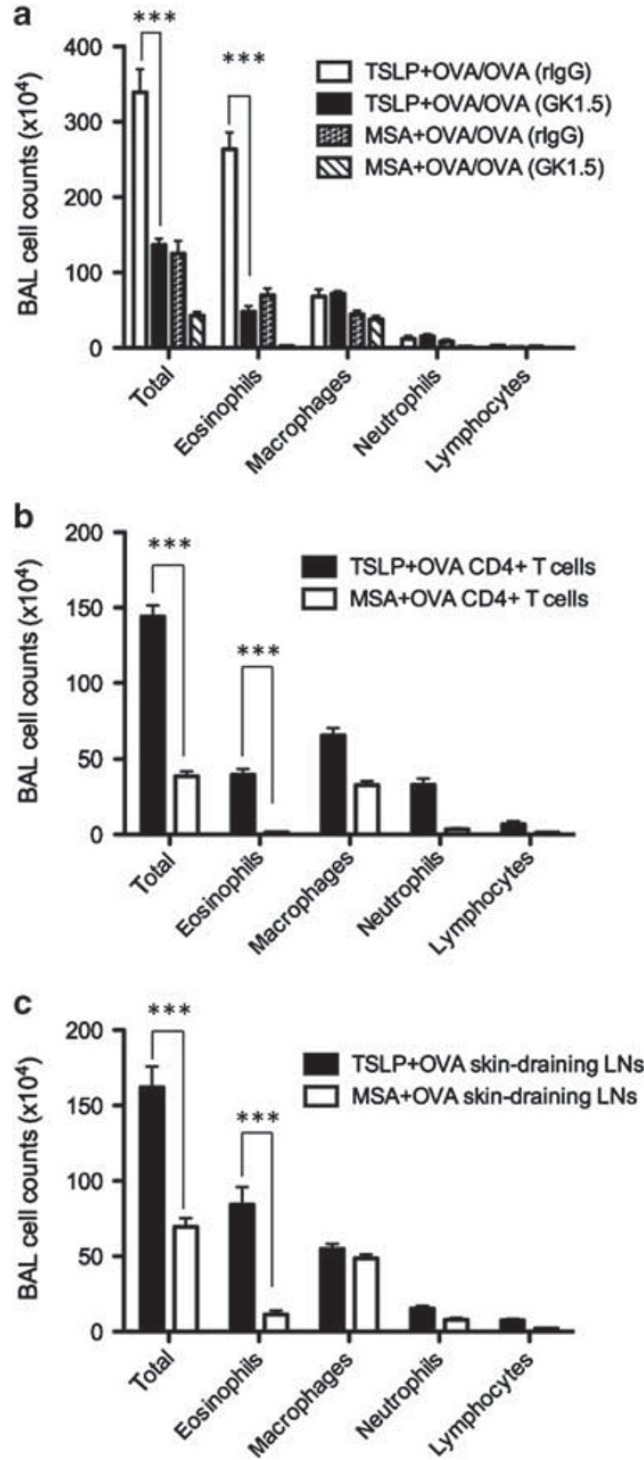

Figure 4 CD4 T cells are required for thymic stromal lymphopoietin (TSLP)-mediated airway inflammation. (a) Cell counts in bronchoalveolar lavage (BAL) fluid from wild-type (WT) BALB/c mice treated intraperitoneally (IP) with rlgG or anti-CD4 antibody (Ab; GK1.5), to acutely deplete CD4 T cells after skin sensitization. $n \geq 4$ mice per group from two independent experiments. (b) DO11.10 CD4 + T cells were transferred to WT BALB/c mice and then sensitized with TSLP+OVA (solid squares) or MSA+OVA (open squares). On day 14 (D14), CD4+ T cells were isolated and negatively selected before transfer of $2 \times 10^{7}$ cells per mouse. Recipient mice were intranasally challenged with ovalbumin (OVA) starting $24 \mathrm{~h}$ after transfer, and airway symptoms were monitored as above. Cell counts in BAL fluid. $n=5$ mice per group. The significance between two groups was determined by two-tailed Student's $t$-test. (c) Skin-draining lymph node (LN) cells from TSLP + OVA-treated mice transfer disease to naïve mice. Skindraining LN cells from mice treated with TSLP + OVA (solid square) and MSA+OVA (open square) were isolated, and cells were cultured with OVA $\left(100 \mathrm{~g} \mathrm{ml}^{-1}\right)$ for $72 \mathrm{~h}$ before washing and intravenous transfer to naive mice ( $2 \times 10^{7}$ cells per mouse). Cell counts in BAL fluid. $n=5$ mice per group. The significance between two groups was determined by two-tailed Student's $t$-test. MSA, mouse serum albumin; OVA, ovalbumin. ${ }^{\star \star \star} P \leq 0.001$.

online, reconstituting mice with DO11.10 CD4 T cells failed to develop the airway inflammatory phenotype in response to intranasal OVA. These data are consistent with a critical role of memory CD4 T cells, primed during skin sensitization, becoming reactivated following intranasal challenge.

We hypothesized that the lack of OVA-specific CD4 + T cells in the airway was responsible for the lack of allergic symptoms. To test this, we first transferred DO11.10 CD4 + T cells to wildtype $\mathrm{BALB} / \mathrm{c}$ mice before skin sensitization. $\mathrm{CD} 4+\mathrm{T}$ cells from primed mice were then isolated and further transferred to naïve recipient mice. Upon intranasal challenge, only mice receiving CD4 + T cells from TSLP + OVA-treated mice developed airway inflammation (Figure 4b). Alternatively, we transferred skindraining lymph node cells from sensitized mice with allergic skin inflammation into naive mice. After OVA challenge of these mice, transfer of the primed cells from TSLP + OVA-treated mice restored airway inflammation (Figure 4c) in recipient mice. Taken together, these data demonstrate a critical role of CD4 + $\mathrm{T}$ cells in the inflammation seen in these mice.

\section{Airway inflammation is antigen specific}

Early exposure to high levels of birch pollen in infancy increases the risk of skin sensitization and allergic asthma to the same allergen. ${ }^{28}$ To test the antigen specificity of our atopic model, mice were sensitized in the skin with TSLP + OVA, and then challenged in the airways with a second antigen, bovine serum albumin (BSA). Control experiments showed that mice sensitized and challenged with BSA responded in a manner similar to that seen with OVA (Figure 5). However, mice that were OVA sensitized and then exposed to intranasal BSA on day 21 displayed a lack of airway inflammation, as judged by total BAL cellularity, eosinophil counts, and antigen-specific IgE (Figure 5a-c). Similarly, BSA-sensitized and OVA-exposed mice were also free of airway inflammation (Figure 5a,b). Similar results were obtained when house dust mice antigen was used as a second antigen (Supplementary Figure S8 online). These data demonstrate that the effect seen in TSLP + antigen-sensitized and -challenged mice was antigen specific.

\section{Tolerance to antigen can be achieved before skin sensitization, but not after}

Previous studies have shown that mice can be tolerized to an aeroantigen through oral administration of the same antigen..$^{29-32} \mathrm{We}$ sought to determine whether oral administration of OVA, either before or after skin sensitization, was capable of tolerizing the mice to subsequent airway exposure to antigen. Mice were given dietary OVA (in the drinking water) for 5 days, either days -7 through -2 before skin sensitization (referred to as Tol -7 ) or days +14 through +19 after the initiation of skin sensitization (referred to as Tol 14). Both untreated and Tol mice were sensitized and challenged as before, and the affect of oral OVA treatment on airway inflammation was determined. As expected from previous studies, the Tol -7 group displayed much-reduced airway inflammation (Figure 6). BAL cellularity was reduced to levels seen in control animals, and both total and antigenspecific IgE were dramatically decreased (Figure 6a,b). In addition, BAL eosinophil numbers were reduced by $>15$-fold, and goblet cell metaplasia, mucus overproduction, as well as overall lung inflammation were markedly decreased (data not shown). 

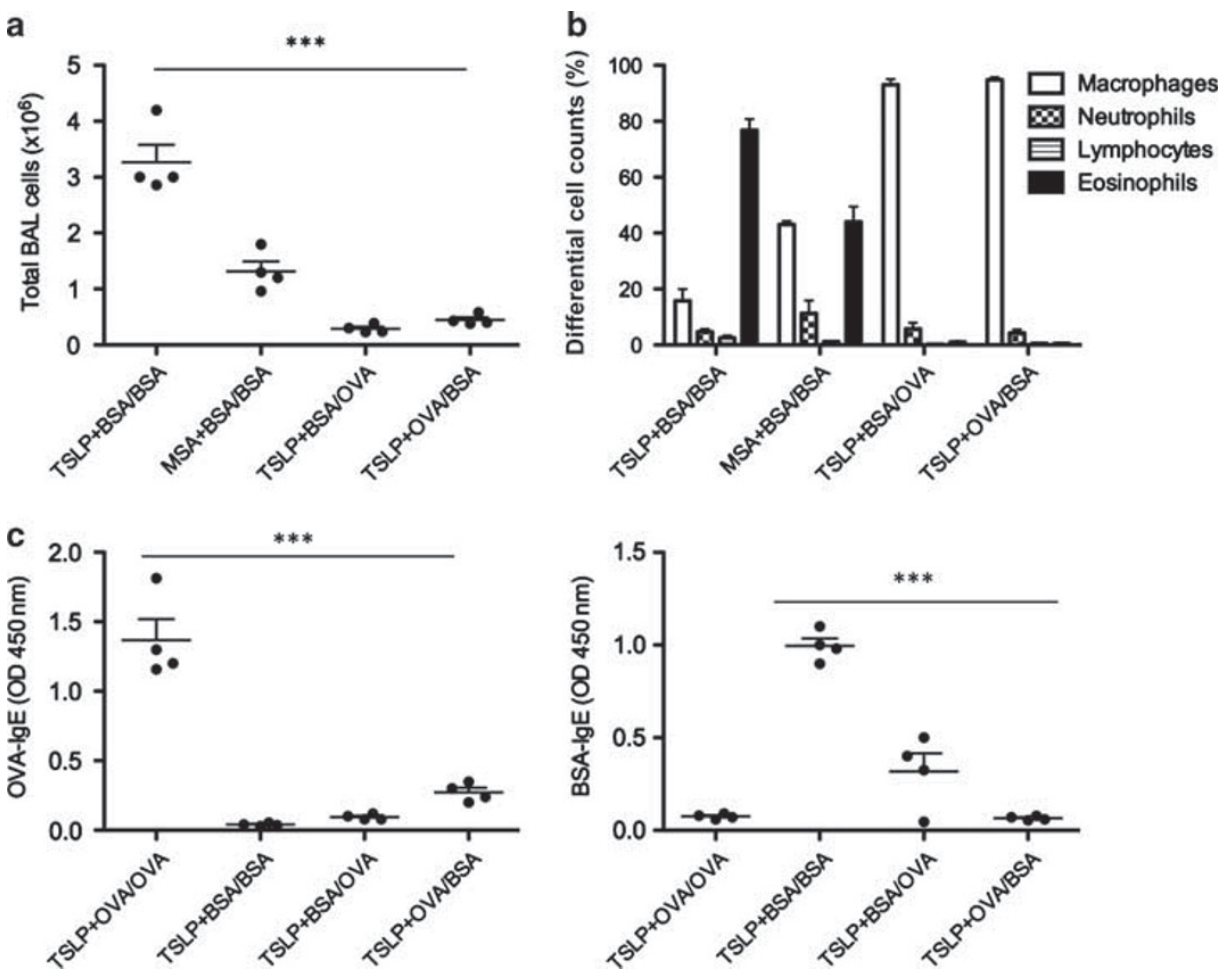

Figure 5 Airway inflammation is antigen specific. To test the antigen specificity of atopic model, low endotoxin bovine serum albumin (BSA) was used to sensitize and challenge the mice. (a) Total cell counts in bronchoalveolar lavage (BAL) fluid. (b) Differential cell counts in BAL. (c) Serum ovalbumin (OVA) and BSA-specific immunoglobulin $\mathrm{E}$ (IgE) on day 25 (D25). $n \geq 4$ mice per group from two independent experiments. MSA, mouse serum albumin; TSLP, thymic stromal lymphopoietin. ${ }^{\star \star *} P \leq 0.001$.

In contrast, the Tol 14 group, when challenged via the airways, displayed an inflammatory response largely equivalent to that seen in the control group (Figure 6). The Tol 14 group showed increased BAL cellularity, lung inflammation, and elevated IgE levels (Figure 6 and data not shown). These data show that, although naive mice can be tolerized to antigen, once sensitized in the presence of TSLP the mice are no longer capable of developing tolerance to that antigen.

\section{Transgenic mice co-overexpressing TSLP and antigen in the skin develop aggravated asthma-like airway inflammation upon challenge}

To study immune responses induced to skin-associated selfantigens, we used mice that inducibly express a TSLP transgene specifically in keratinocytes in the presence of dietary doxycycline. ${ }^{13}$ These mice were crossed to mice that contain a similar transgene that expresses a membrane-bound form of OVA, using the same regulatory mechanisms as the K5-TSLP mice (see Methods for details of the mouse strains). We generated triple-transgenic mice (referred to as K5-TSLP/TGO), allowing keratinocyte-specific expression of both transgenes in the presence of doxycycline. We first monitored proliferation of OVA-specific CD4 T cells following transfer into doxycyclinetreated K5-TGO mice. Following transfer, robust proliferation was seen in skin-draining lymph, with much less seen in more distal sites (Figure 7a). No proliferation was observed when cells were transferred into control mice. K5-TSLP/TGO mice were then given dietary doxycycline $\left(1 \mathrm{mg} \mathrm{ml}^{-1}\right.$ for 3 weeks $)$ to induce
TSLP and OVA expression in keratinocytes. To exclude a role for circulating TSLP affecting responses during the skin sensitization phase, mice were treated intranasally with the anti-mTSLP antibody described above on D0 and D12. At the end of the doxycyline treatment period, the skin of the K5-TSLP/TGO mice displayed features consistent with eczematous inflammation, ${ }^{13}$ which subsequent receded and ultimately disappeared during the rest period. These features were not present in doxycyclinetreated K5-TGO skin. Following an 18-day rest period, the mice were treated intranasally with OVA for four consecutive days. Only the K5-TSLP/TGO mice developed airway inflammation (Figure 7b,c). Thus, the induced expression of epidermal TSLP with OVA was sufficient to drive an aggravation of the asthma that was generated upon OVA challenge at a time when the skin $\mathrm{AD}$ had resolved.

\section{DISCUSSION}

Allergic diseases tend to develop in a sequential manner in childhood, a phenomenon referred to as the "atopic march."33 A wide variety of epidemiological and birth cohort studies have been performed to attempt to explain this. For example, this phenomenon commonly manifests as a child with $\mathrm{AD}$ going on to subsequently develop asthma, or allergic reactions to aeroallergens. ${ }^{34}$ This may be because of the fact that, of the atopic diseases, $\mathrm{AD}$ seems to develop earliest, with $85 \%$ of those individuals with the disease becoming symptomatic by age $5 .{ }^{35}$ This phenomenon has suggested that these diseases share a common underlying mechanism or factor, capable of promoting a 


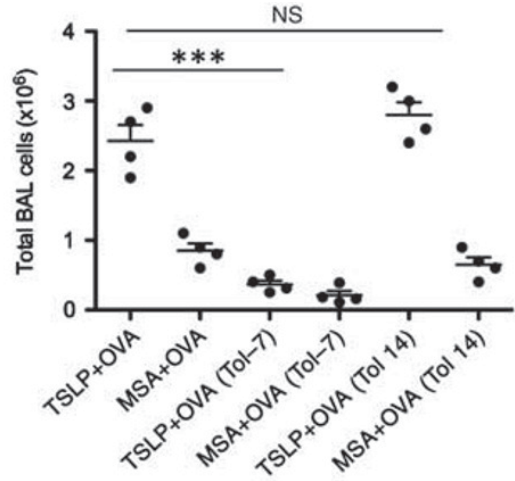

b

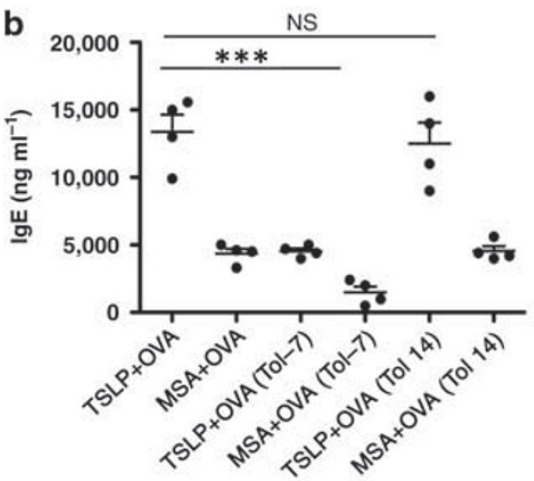

C

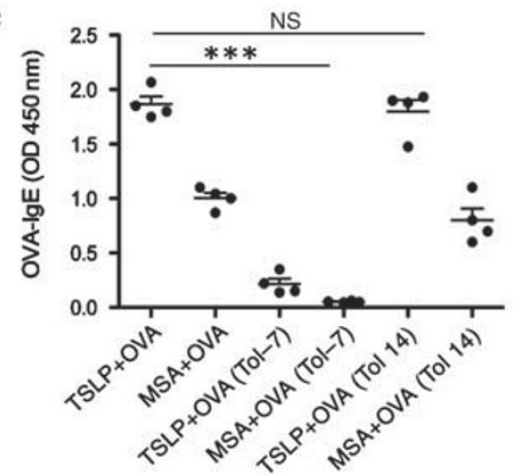

Figure 6 Antigen feeding before, but not after, skin sensitization suppresses asthma-like inflammatory response. Mice were administered a $1 \%$ ovalbumin (OVA) solution in drinking water for 5 consecutive days (days -7 to -2 , Tol -7 ; or days $14-19$, Tol 14). (a) Cell counts in bronchoalveolar lavage (BAL) fluid. (b) Total and (c) OVA-specific serum immunoglobulin $E$ (IgE) on day 25 (D25). $n=4$ mice per group from two independent experiments. MSA, mouse serum albumin; NS, not significant; TSLP, thymic stromal lymphopoietin. ${ }^{* \star *} P \leq 0.001$.

Th2-type response to environmental antigens. One candidate for this factor is TSLP, which is overexpressed in the affected tissues in each of these diseases. ${ }^{8,12,36}$ The work presented herein supports this model, and demonstrates that sensitization to antigen, in the presence of TSLP, in the skin leads to aggravated pulmonary responses when that antigen is inhaled.

Recently, two studies have shown a link between elevated TSLP expression in the skin and airway inflammation following aero-antigen challenge. ${ }^{18,19}$ Similarly, we have found that, in addition to skin inflammation, doxycycline-treated K5-TSLP mice develop pulmonary inflammation similar to the SPC-TSLP mice after $>5$ weeks of treatment (Supplementary Figure S1 online). The major caveat with each of these systems is that, unlike human $\mathrm{AD}$ patients, these mice all displayed elevated circulating levels of TSLP (Figure 2 and refs. 18 and 19). Thus, it is not possible to distinguish between an effect of circulating TSLP-promoting responses directly in the lung or an alteration of responses in the lung subsequent to TSLP effects locally in the skin. In addition to pulmonary inflammation, these three mouse models also develop a variety of inflammatory responses at other sites, further complicating the interpretation of the data. ${ }^{10,19,37}$ To circumvent these caveats, we have developed a mouse model that uses intradermal injection of TSLP, in the presence or absence of antigen, followed by intranasal challenge after a rest period (see Figure 1 for schematic of model). As shown in Figure 1, these mice develop significant airway inflammation following intranasal challenge. Importantly, they do not have detectable circulating levels of TSLP. For these reasons, we feel that this model more faithfully represents the events involved in the atopic march.

Epicutaneous sensitization with allergen followed by airway challenge induces airway inflammation and airway hyperresponsiveness. ${ }^{38,39}$ Our data provide strong evidence that cutaneous exposure to allergen, in the presence of TSLP, can trigger progression from $\mathrm{AD}$ to asthma. Using intradermal administration of TSLP, we found that mice sensitized to an antigen in this fashion develop allergic airway inflammation when subsequently challenged via the airways with the same antigen. This indicates that allergen sensitization in the skin can affect and sensitize the airways. Interestingly, our data indicate that TSLP is only involved in the sensitization phase of the response, whereas the subsequent response to pulmonary challenge is TSLP independent. This suggests that TSLP is acting locally to drive the initial response to allergen challenge, and that the subsequent "march" of the allergic response to the lung is regulated by signals delivered during this cutaneous response. Although the factors that mediate the lung pathology following intranasal challenge remain to be elucidated, our data demonstrate that CD4 memory $\mathrm{T}$ cells are required for airway inflammation. Administration of naive, antigen-specific cells following skin sensitization and CD4 depletion failed to reconstitute the pulmonary response following antigen challenge.

TSLP is one of the several epithelial-derived cytokines capable of promoting type-2 immune responses. ${ }^{21}$ In particular, IL-25 expression, like TSLP, is elevated in lesional skin of AD patients. ${ }^{22,40}$ It has also been shown to promote Th2-type responses through the expansion of skin-homing Th2 memory cells. ${ }^{22}$ It was of note, therefore, that cutaneous administration of antigen with IL-25 failed to promote airway inflammation upon subsequent pulmonary antigen challenge (Supplementary Figure $\mathbf{S 4}$ online). These data suggest that the response seen in mice sensitized with antigen and TSLP is specific for TSLP, and not simply a general promotion of a Th2 response.

Finally, the observation that once sensitized to antigen in the presence of TSLP, mice cannot be tolerized to that antigen is of significance and has implications for the development of treatments for individuals who develop asthma subsequent to $\mathrm{AD}$. As, before skin immunization, naïve OVA-specific $\mathrm{T}$ cells that will become regulatory $\mathrm{T}$ cells cannot be distinguished from those 

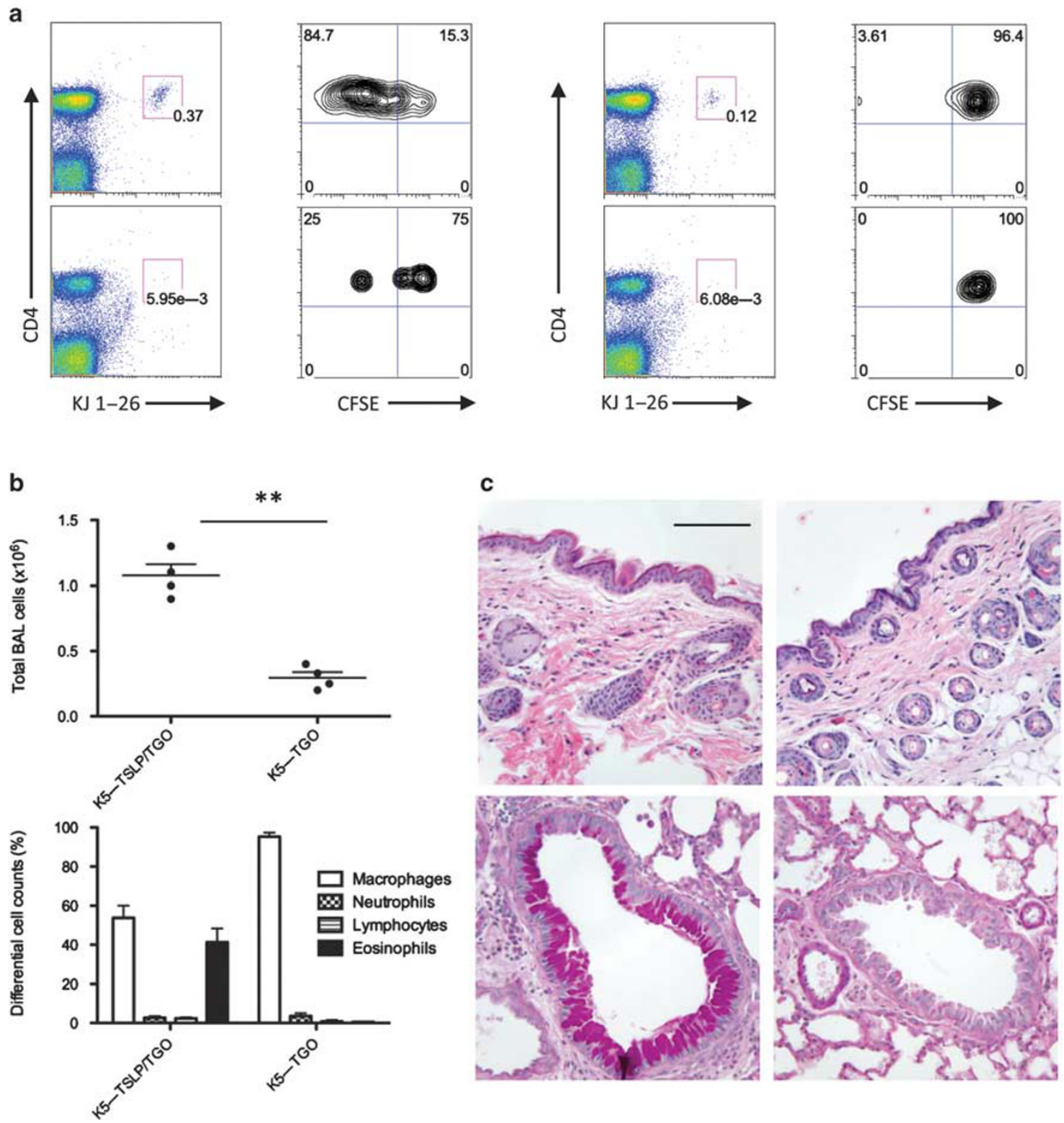

Figure 7 Mice overexpressing thymic stromal lymphopoietin (TSLP) and antigen in the skin develop aggravated asthma-like airway inflammation. (a) Ovalbumin (OVA)-specific T cells are primed in K5-TGO mice. K5-TGO mice received $1 \mathrm{mg} \mathrm{ml}^{-1}$ of doxycycline for 3 weeks of treatment. Profile of DO11.10 CD4 T cells transferred into K5-TGO (left) and control (right) mice were determined in inguinal lymph node (upper) and spleen (lower). (b) Total cell counts and differential cell counts in the bronchoalveolar lavage (BAL) $(n=4)$. (c) Hematoxylin and eosin (H\&E) staining of skin (upper) and periodic acid-Schiff (PAS) staining of lung (lower) from K5-TSLP/TGO mice (left) and K5-TGO mice (right). CFSE, carboxyfluorescein diacetate succinimidyl ester. Bar $=100 \mu \mathrm{m} .{ }^{\star \star} P \leq 0.01$.

that will become Th2 cells, it remains to be determined whether generation of regulatory $\mathrm{T}$ cells vs. that of effector $\mathrm{T}$ cells is a stochastic process or some microenvironmental conditions promote the development of one cell type over the other. Therapies that modify the severity of AD in infants and young children might decrease the risk for the eventual development of asthma.

In conclusion, our present study not only provides a mouse model for studying possible mechanisms involved in the "atopic march," but also suggests that overproduction of TSLP in the skin of patients with AD could be a risk factor for the development of allergic airway inflammation. Together, our study supports the hypothesis that skin may be an important site for systemic sensitization to certain allergens, and that epidermal TSLP may promote sensitization, asthma, and food allergies. Collectively, the results of our study elucidate a novel mechanism for the atopic march, and implicate TSLP as an initiating factor in this disease process.

\section{METHODS}

An expanded Materials and Methods section is available in the Data Supplement online.

Mice and injections. Female BALB/c mice, 6-8 weeks old, were obtained from Charles River Laboratories (Wilmington, MA). K5-TSLP mice were generated as described previously. ${ }^{10,13} \mathrm{C} 3 \mathrm{H} / \mathrm{Hej}-\mathrm{BALB} / \mathrm{c}$ (TLR4mut) 
and DO11.10 mice were purchased from The Jackson Laboratory (Bar Harbor, ME) and described previously. ${ }^{41}$ All animals were housed in specific pathogen-free conditions in the Benaroya Research Institute (Seattle, WA) animal facility. Intradermal injections were performed, as previously described, ${ }^{20}$ with minimal modifications to the dose volume. Briefly, fur was stripped from the lower backs of mice, and $5 \mu \mathrm{g}$ TSLP (generous gift of Amgen, Seattle, WA) or MSA (Sigma-Aldrich, St Louis, MO) with OVA (A7642; Sigma-Aldrich), low endotoxin BSA (SigmaAldrich), or house dust mice antigen (Greer Laboratories, Lenoir, NC) were injected intradermally in a $100 \mu \mathrm{l}$ volume of sterile phosphatebuffered saline by inserting the needle into the superficial dermis as close to the epithelium as possible. Experimental protocols were approved by the institutional animal care and use committees of Benaroya Research Institute. Detailed information on the generation of TRE-TGO and TSLP-deficient mice is in the Data Supplement online.

TSLP antibody treatment. To block TSLP in the lung at the time of skin sensitization, an antibody to murine TSLP (R\&D Systems) was administered intranasally ( $50 \mu \mathrm{g}$ intranasally on D0 and D8). Acute blocking of TSLP after skin sensitization was performed as previously described, ${ }^{14}$ with minimal modifications. In brief, mice were treated with TSLP blocking antibody on days 14 (200 $\mu$ g intraperitoneally) and $15(100 \mu \mathrm{g}$ intravenously). Mice received further treatments on days $19(100 \mu \mathrm{g}$ intravenously) and 20 (50 $\mu$ g intranasally) followed by OVA challenge.

Statistics. All statistical analyses were performed using GraphPad Prism 5 (San Diego, CA). Unless otherwise indicated, all statistical tests are oneway analysis of variance with Tukey's post-hoc test, with significance between groups represented as ${ }^{\star} P \leq 0.05,{ }^{* *} P \leq 0.01$, and ${ }^{* *} P \leq 0.001$.

SUPPLEMENTARY MATERIAL is linked to the online version of the paper at http://www.nature.com/mi

\section{ACKNOWLEDGMENTS}

We thank Drs Jessica Hamerman, Daniel J. Campbell, and Mark Schlomchik for critical discussions and review of the manuscript. We thank Matt Warren for his administrative support. This work was partially supported by the NIH Grants Al68731, AR55695, and AR56113 to S.F.Z, and CA90691 to A.M.-R.

\section{DISCLOSURE}

The authors declared no conflict of interest.

(c) 2012 Society for Mucosal Immunology

\section{REFERENCES}

1. Bieber, T. Atopic dermatitis. N. Engl. J. Med. 358, 1483-1494 (2008).

2. Spergel, J.M. \& Paller, A.S. Atopic dermatitis and the atopic march. J. Allergy Clin. Immunol. 112, S118-S127 (2003).

3. Beck, L.A. \& Leung, D.Y. Allergen sensitization through the skin induces systemic allergic responses. J. Allergy Clin. Immunol. 106, S258-S263 (2000).

4. Friend, S.L. et al. A thymic stromal cell line supports in vitro development of surface lgM+ B cells and produces a novel growth factor affecting B and T lineage cells. Exp. Hematol. 22, 321-328 (1994).

5. Sims, J.E. et al. Molecular cloning and biological characterization of a novel murine lymphoid growth factor. J. Exp. Med. 192, 671-680 (2000).

6. Liu, Y.J. et al. TSLP: an epithelial cell cytokine that regulates T cell differentiation by conditioning dendritic cell maturation. Annu. Rev. Immunol. 25, 193-219 (2007).

7. Reche, P.A. et al. Human thymic stromal lymphopoietin preferentially stimulates myeloid cells. J. Immunol. 167, 336-343 (2001).

8. Soumelis, V. et al. Human epithelial cells trigger dendritic cell mediated allergic inflammation by producing TSLP. Nat. Immunol. 3, 673-680 (2002).

9. Ziegler, S.F. \& Liu, Y.J. Thymic stromal lymphopoietin in normal and pathogenic T cell development and function. Nat. Immunol. 7, 709-714 (2006).
10. Astrakhan, A. et al. Local increase in thymic stromal lymphopoietin induces systemic alterations in B cell development. Nat. Immunol. 8, 522-531 (2007).

11. Ray, R.J., Furlonger, C., Williams, D.E. \& Paige, C.J. Characterization of thymic stromal-derived lymphopoietin (TSLP) in murine B cell development in vitro. Eur. J. Immunol. 26, 10-16 (1996).

12. Ying, S. et al. Thymic stromal lymphopoietin expression is increased in asthmatic airways and correlates with expression of Th2-attracting chemokines and disease severity. J. Immunol. 174, 8183-8190 (2005).

13. Yoo, J. et al. Spontaneous atopic dermatitis in mice expressing an inducible thymic stromal lymphopoietin transgene specifically in the skin. J. Exp. Med. 202, 541-549 (2005).

14. Larson, R.P. et al. Dibutyl phthalate-induced thymic stromal lymphopoietin is required for Th2 contact hypersensitivity responses. J. Immunol. 184, 2974-2984 (2010)

15. Zhou, B. et al. Thymic stromal lymphopoietin as a key initiator of allergic airway inflammation in mice. Nat. Immunol. 6, 1047-1053 (2005).

16. Al-Shami, A., Spolski, R., Kelly, J., Keane-Myers, A. \& Leonard, W.J. A role for TSLP in the development of inflammation in an asthma model. J. Exp. Med. 202, 829-839 (2005).

17. Cookson, W. The immunogenetics of asthma and eczema: a new focus on the epithelium. Nat. Rev. Immunol. 4, 978-988 (2004).

18. Zhang, Z. et al. Thymic stromal lymphopoietin overproduced by keratinocytes in mouse skin aggravates experimental asthma. Proc. Natl. Acad. Sci. USA 106, 1536-1541 (2009).

19. Demehri, S., Morimoto, M., Holtzman, M.J. \& Kopan, R. Skin-derived TSLP triggers progression from epidermal-barrier defects to asthma. PLoS Biol. 7, e1000067 (2009).

20. Jessup, H.K. et al. Intradermal administration of thymic stromal lymphopoietin induces a T cell- and eosinophil-dependent systemic Th2 inflammatory response. J. Immunol. 181, 4311-4319 (2008).

21. Saenz, S.A., Taylor, B.C. \& Artis, D. Welcome to the neighborhood: epithelial cell-derived cytokines license innate and adaptive immune responses at mucosal sites. Immunol. Rev. 226, 172-190 (2008).

22. Wang, Y.H. et al. IL-25 augments type 2 immune responses by enhancing the expansion and functions of TSLP-DC-activated Th2 memory cells. J. Exp. Med. 204, 1837-1847 (2007).

23. Nakajima, H. et al. CD4+ T-lymphocytes and interleukin-5 mediate antigen-induced eosinophil infiltration into the mouse trachea. Am. Rev. Respir. Dis. 146, 374-377 (1992).

24. Gavett, S.H., Chen, X., Finkelman, F. \& Wills-Karp, M. Depletion of murine CD4+ T lymphocytes prevents antigen-induced airway hyperreactivity and pulmonary eosinophilia. Am. J. Respir. Cell Mol. Biol. 10, 587-593 (1994).

25. Brusselle, G.G. et al. Attenuation of allergic airway inflammation in IL-4 deficient mice. Clin. Exp. Allergy 24, 73-80 (1994).

26. Cohn, L., Tepper, J.S. \& Bottomly, K. IL-4-independent induction of airway hyperresponsiveness by Th2, but not Th1, cells. J. Immunol. 161, 3813-3816 (1998).

27. Herrick, C.A. \& Bottomly, K. To respond or not to respond: T cells in allergic asthma. Nat. Rev. Immunol. 3, 405-412 (2003).

28. Kihlstrom, A., Lilja, G., Pershagen, G. \& Hedlin, G. Exposure to birch pollen in infancy and development of atopic disease in childhood. J. Allergy Clin. Immunol. 110, 78-84 (2002).

29. Mucida, D. et al. Oral tolerance in the absence of naturally occurring Tregs. J. Clin. Invest 115, 1923-1933 (2005).

30. Zhang, X., Izikson, L., Liu, L. \& Weiner, H.L. Activation of CD25(+)CD4(+) regulatory T cells by oral antigen administration. J. Immunol. 167, 4245-4253 (2001).

31. Hauet-Broere, F., Unger, W.W., Garssen, J., Hoijer, M.A., Kraal, G. \& Samson, J.N. Functional CD25- and CD25+ mucosal regulatory T cells are induced in gut-draining lymphoid tissue within $48 \mathrm{~h}$ after oral antigen application. Eur. J. Immunol. 33, 2801-2810 (2003).

32. Faria, A.M. et al. Oral tolerance induced by continuous feeding: enhanced up-regulation of transforming growth factor-beta/interleukin-10 and suppression of experimental autoimmune encephalomyelitis. J. Autoimmun. 20, 135-145 (2003).

33. Hahn, E.L. \& Bacharier, L.B. The atopic march: the pattern of allergic disease development in childhood. Immunol. Allergy Clin. North Am. 25, 231-46, v (2005).

34. Kulig, M., Bergmann, R., Niggemann, B., Burow, G. \& Wahn, U. Prediction of sensitization to inhalant allergens in childhood: evaluating 
family history, atopic dermatitis and sensitization to food allergens. The MAS Study Group. Multicentre Allergy Study. Clin. Exp. Allergy 28, 1397-1403 (1998).

35. Kay, J., Gawkrodger, D.J., Mortimer, M.J. \& Jaron, A.G. The prevalence of childhood atopic eczema in a general population. J. Am. Acad. Dermatol. 30, 35-39 (1994).

36. Mou, Z. et al. Overexpression of thymic stromal lymphopoietin in allergic rhinitis. Acta Otolaryngol. 129, 297-301 (2009).

37. Li, M. et al. Topical vitamin D3 and low-calcemic analogs induce thymic stromal lymphopoietin in mouse keratinocytes and trigger an atopic dermatitis. Proc. Natl. Acad. Sci. USA 103, 11736-11741 (2006).
38. Spergel, J.M. et al. Epicutaneous sensitization with protein antigen induces localized allergic dermatitis and hyperresponsiveness to methacholine after single exposure to aerosolized antigen in mice. J. Clin. Invest. 101, 1614-1622 (1998).

39. He, R. et al. Exaggerated IL-17 response to epicutaneous sensitization mediates airway inflammation in the absence of IL-4 and IL-13. J. Allergy Clin. Immunol. 124, 761-770 (2009).

40. Hvid, M. et al. IL-25 in atopic dermatitis: a possible link between inflammation and skin barrier dysfunction? J. Invest. Dermatol. 131, 150-157 (2011)

41. Headley, M.B. et al. TSLP conditions the lung immune environment for the generation of pathogenic innate and antigen-specific adaptive immune responses. J. Immunol. 182, 1641-1647 (2009). 